\title{
A SHORT PROOF OF THE FACT THAT THE MATRIX TRACE IS THE EXPECTATION OF THE NUMERICAL VALUES
}

\author{
TOMASZ KANIA
}

\begin{abstract}
Using the fact that the normalised matrix trace is the unique linear functional $f$ on the algebra of $n \times n$ matrices which satisfies $f(I)=1$ and $f(A B)=f(B A)$ for all $n \times n$ matrices $A$ and $B$, we derive a well-known formula expressing the normalised trace of a complex matrix $A$ as the expectation of the numerical values of $A$; that is the function $\langle A x, x\rangle$, where $x$ ranges the unit sphere of $\mathbb{C}^{n}$.
\end{abstract}

Let $A=\left[a_{i j}\right]$ be an $n \times n$ complex matrix. The aim of this note is to give an easy proof of the fact that the normalised trace of $A, \operatorname{tr} A=\frac{1}{n}\left(a_{11}+a_{22}+\ldots+a_{n n}\right)$, can be thought of as the expectation of the numerical values of $A$; that is the function $x \mapsto\langle A x, x\rangle$ defined on the Euclidean unit sphere in $\mathbb{C}^{n}$, endowed with the normalised Lebesgue surface measure $\mu$. More precisely,

$$
\operatorname{tr} A=\int_{\|x\|=1}\langle A x, x\rangle \mu(\mathrm{d} x) .
$$

The above formula is a particular version of a more general identity for symmetric 2-tensors on Riemannian manifolds (consult e.g. [2] see also [1] for the proofi). We offer here an elementary proof of Equation (1) relying on two folklore facts from linear algebra.

Lemma 1. The matrix trace is unique in the sense that it is the unique linear functional $f: M_{n}(\mathbb{C}) \rightarrow \mathbb{C}$ satisfying the following properties:

(i) $f(I)=1$, where $I$ denotes the $n \times n$ identity matrix,

(ii) $f(A B)=f(B A)$ for all $A, B \in M_{n}(\mathbb{C})$.

It is evident that the standard normalised trace $\operatorname{tr}$ on $M_{n}(\mathbb{C})$ satisfies conditions (ii)-(ii), so in order to prove the above lemma, it is enough to show that a functional $f$ enjoying (ii)-(iii) agrees with $\operatorname{tr}$ on the standard matrix units $e_{i j}=\left[\delta_{i, j}\right](1 \leqslant i, j \leqslant n)$; that is, $f\left(e_{i j}\right)=\frac{1}{n}$ whenever $i=j$ and $f\left(e_{i j}\right)=0$ otherwise. We leave this as an exercise for the reader.

We shall require also the following easy and well-known fact. (See also [3, Lemma $3.2 .21]$.

Lemma 2. Every complex $n \times n$ matrix is a linear combination of unitary matrices.

Proof. Every matrix $A \in M_{n}(\mathbb{C})$ can be written as a linear combination of two self-adjoint matrices, so without loss of generality it is enough to show that each self-adjoint matrix

\footnotetext{
${ }^{1}$ Bennett Chow blames one of his students for a neat proof he posted at [1].
} 
$A$ with $\|A\| \leqslant 1$ can be written as a linear combination of unitaries. To this end, set $U=A-i\left(I-A^{2}\right)^{\frac{1}{2}}$ and note that $U$ is unitary. Clearly $A=\frac{1}{2} U+\frac{1}{2} U^{*}$.

We are now in a position to prove Equation (1).

Proof. Let $f(A)$ denote the right hand side of Equation (1). It is enough to verify that $f$ meets conditions (ii)-(ii) of Lemma 1. Evidently, $f$ is linear, and $f(I)=1$ because $\mu$ is a probability measure. It remains to show that (iii) holds.

Let $A, B \in M_{n}(\mathbb{C})$ and let us write $B$ as a linear combination of some unitary matrices $U_{1}, \ldots, U_{m}$, that is $B=\sum_{k=1}^{m} a_{k} U_{k}$ for some scalars $a_{1}, \ldots, a_{m}$. We may assume additionally that each matrix $U_{k}(k \leqslant n)$ has determinant 1 , as we can always write $B=\sum_{k=1}^{m}\left(a_{k} \operatorname{det} U_{k}\right) \frac{U_{k}}{\operatorname{det} U_{k}}$. We have $f(A B)=f(B A)$ as soon as $f\left(A U_{k}\right)=f\left(U_{k} A\right)$ for all $k \leqslant m$, so that without lost of generality we may suppose that $B$ is unitary and $\operatorname{det} B=1$. Making the substitution $x=B^{*} z$ and taking into account that the determinant of $B$ is equal to 1 (hence also the Jacobian of $B$, regarded as a map from the real $(2 n-1)$-sphere to itself, is equal to 1 ), we arrive at the conclusion that

$$
\begin{aligned}
f(A B) & =\int_{\|x\|=1}\langle A B x, x\rangle \mu(\mathrm{d} x) \\
& =\int_{\|x\|=1}\left\langle A z, B^{*} z\right\rangle \mu(\mathrm{d} z) \\
& =\int_{\|x\|=1}\langle B A z, z\rangle \mu(\mathrm{d} z) \\
& =f(B A),
\end{aligned}
$$

which completes the proof.

\section{REFERENCES}

1. B. Chow, Geometric interpretation of tracing (2013), available at http://mathoverflow.net/questions/127692/geometric-interpretation-of-tracing/150260\#150260.

2. S. Gallot, D. Hulin, J. Lafontaine, Riemannian geometry. Universitext, Springer-Verlag, Berlin, Heidelberg, New York, 1987.

3. G. K. Pedersen, Analysis Now, Springer-Verlag, New York, 1989.

Department of Mathematics and Statistics, Fylde College, Lancaster University, LanCASTER LA1 4YF, UNITED KINGDOM

Current address: Institute of Mathematics, Polish Academy of Sciences, Śniadeckich 8, 00-956 WARsZaWA, POLAND

E-mail address: tomasz.marcin.kania@gmail.com 